\title{
Titanium carbide nanoparticles/ion-exchange polymer-based sensor for catalytic stripping determination of trace iron in coastal waters
}

\author{
Mingyue Lin ${ }^{\mathrm{a}, \mathrm{b}}$, Dawei Pan ${ }^{\mathrm{a}, *}$, Xueping Hu ${ }^{\mathrm{a}, \mathrm{b}}$, Haitao Han ${ }^{\mathrm{a}}$, Fei Li ${ }^{\mathrm{c}}$ \\ ${ }^{a}$ Key Laboratory of Coastal Environmental Processes and Ecological Remediation, Yantai Institute of Coastal Zone Research (YIC), Chinese Academy of \\ Sciences (CAS); Shandong Provincial Key Laboratory of Coastal Environmental Processes, YICCAS, Yantai, Shandong 264003, PR China \\ ${ }^{\mathrm{b}}$ University of Chinese Academy of Sciences, Beijing 100049, PR China \\ ${ }^{\mathrm{c}}$ The Key Lab in Molecular and Nano-materials Probes of the Ministry of Education of China, College of Chemistry, Chemical Engineering and Materials \\ Science, Shandong Normal University, Jinan, Shandong 250014, PR China
}

\section{A R T I C L E I N F O}

\section{Article history:}

Received 18 March 2015

Received in revised form 4 May 2015

Accepted 14 May 2015

Available online 22 May 2015

\section{Keywords:}

Iron

TiC nanoparticles

Nafion

Catalytic stripping voltammetry

Coastal waters

\begin{abstract}
A B S T R A C T
We establish a novel nanoparticle/ion-exchange polymer-based sensor for selective and sensitive detection of trace iron $\left(\mathrm{Fe}^{3+}\right)$ in coastal waters. Titanium carbide nanoparticles (nano-TiC) are used as a typical nanomaterial with promising physical and chemical properties to accelerate the electron transfer. Nafion is chosen as the widely used cation-exchange polymer to facilitate the preconcentration of $\mathrm{Fe}^{3+}$. Taking advantage of synergistic effects of nano-TiC and Nafion as well as the catalytic amplifying effect of hydrogen peroxide $\left(\mathrm{H}_{2} \mathrm{O}_{2}\right)$, the excellent cathodic signal responses for the stripping determination of $\mathrm{Fe}^{3+}$ which is linear in the range of $0.07-70 \mu \mathrm{M}$ with a detection limit of $7.2 \mathrm{nM}$ can be obtained. This analytical method can be used to effectively and simply detect $\mathrm{Fe}^{3+}$ without using any complexing agents. The fabricated sensor has been successfully applied for the sensitive determination of $\mathrm{Fe}^{3+}$ in real coastal waters.
\end{abstract}

(C) 2015 Elsevier B.V. All rights reserved.

\section{Introduction}

Iron is one of the most common elements in the earth's crust. As one of the essential micronutrients, iron is involved in the plant metabolism where it is pivotal for photosynthetic and respiratory electron transport, nitrate reduction, and chlorophyll synthesis [1]. According to iron limitation hypothesis [2], its existence in the environment is of major interest due to its important role in oceanic biogeochemical processes [3]. In addition, it has been shown that iron also plays an important role in nutrient cycling processes such as nitrogen fixation and can limit growth in non-HNLC (highnutrient, low chlorophyll) regions [4] and coastal upwelling areas [5-7], and may have a connection with red tide [8]. It has been reported that the concentration of dissolved iron in coastal water is greater than that in seawater and the iron cycle in coastal zones is more complex due to the proximity to terrestrial, continental shelf sources and human activity [9]. Therefore, the accurate and simple methods for the detection of iron in coastal waters are very eager to be developed.

\footnotetext{
* Corresponding author. Tel.: +86 535 2109155; fax: +86 5352109155

E-mail address: dwpan@yic.ac.cn (D. Pan).
}

Among a variety of new analytical tools under development, electrochemical method has been recognized as one the most sensitive methods for trace iron analysis and it possesses the advantages of low cost, simple and convenient operation, fast experimental process, high sensitivity and selectivity, as well as potential application for in situ experiments or on-site monitoring [10]. Traditionally, the most sensitive electrochemical methods for iron determination were based on hanging drop mercury electrodes by stripping voltammetry with the aid of complexing agents to lower the detection limit which required complicated procedures such as pre-complexation, pH adjustment, and so forth [11,12]. Considering the drawbacks of mercury electrode, such as toxicity, difficulties in handling, storage and disposal as well as the complicated procedures in pretreatment, it may severely restrict its application [13]. In order to solve these problems, great efforts have been taken to develop mercury-free solid electrodes. For example, solid-state gold-amalgamation [14], ion-selective membranes [15], ionomercoatings [16-19], carbon paste [20], glassy carbon [21] electrodes and gold microelectrode ensembles [22] as new solid electrodes have been developed for iron determination. However, the continuous improvements of functional electrodes are still urgently required for achieving simple, rapid and sensitive detection of trace iron, especially in coastal waters. 
In order to increase the selectivity and sensitivity of the electrode, current works mainly focus on the chemical modification of electrode surface [23]. Polymers which can form a thin film on a solid electrode surface are usually utilized for the preconcentration of target and play an important role in trace environmental analyses $[24,25]$. Nafion as one of the most widely used cation-exchange polymer possesses the ideal electrochemical properties as well as the high chemical and mechanical stability. In previous reports, Nafion has shown benefits in facilitating the preconcentration of $\mathrm{Fe}^{3+}, \mathrm{Fe}^{2+}$ and iron complexes [16-19]. Except for the selectivity, the acceleration of electron transfer rate is also an important aspect to be considered. The application of nanotechnology has become one of the most active areas in analytic chemistry. Nanomaterials of various shapes, sizes, and compositions have been widely used for the construction and modification of electrochemical sensors, because they possess unique electronic conductivity, and large specific surface areas [26,27]. Nanomaterials, such as gold nanoparticles [28], multiwall carbon nanotubes [29], nanocomposite of reduced graphene oxide, methylene blue, and gold nanoparticles [30] have been successfully used for iron determinations. Nowadays, nanosized transition metal carbides, particularly titanium carbide nanoparticles (nano-TiC) have attracted significant interest for a number of applications in analytical chemistry due to its intrinsic material properties such as high electrical conductivity, low density, high surface area and catalytic activity [31-33]. Moreover, nano-TiC shows greater advantages and novel characteristics than regular sized particles, such as larger specific surface area and high electron transfer rate [34]. Besides, its cubic phase exhibits interesting photocatalytic and electrocatalytic behaviors [35-37], which has been used as the electrode material for catalytic determination of $\mathrm{H}_{2} \mathrm{O}_{2}$ [34].

The aim of this paper is to combine the unique properties of nanomaterials with the specific preconcentration ability of ionexchange polymer to fabricate an electrochemical sensing platform toward $\mathrm{Fe}^{3+}$ determination. Based on the synergistic effects of nano-TiC and Nafion, as well as the help of the catalytic amplifying effect of $\mathrm{H}_{2} \mathrm{O}_{2}$, such a designed nano-TiC/Nafion modified electrode can offer remarkably improved sensitivity and selectivity for voltammetric measurement of $\mathrm{Fe}^{3+}$. Experimental conditions and analytical performances were systematically investigated. The developed sensor was also successfully applied for the detection of total dissolved iron in real coastal waters with satisfactory results.

\section{Experimental}

\subsection{Reagents}

Unless stated otherwise, all chemicals used are analytical reagent grade. Deionized water $(18.2 \mathrm{M} \Omega \mathrm{cm}$ specific resistance) from Pall Cascada laboratory water system was used throughout to prepare the solutions. $\mathrm{HNO}_{3}$ and $\mathrm{HCl}$ were Guaranteed Reagents and were sub-boiling distilled using a quartz condenser. Iron standard solutions were prepared from chloride of $\mathrm{Fe}^{3+}$ (Sinopharm Chemical Reagent Co., Ltd., China.) in $0.1 \mathrm{M} \mathrm{HCl}$. $\mathrm{HNO}_{3}$, $\mathrm{HCl}, \mathrm{H}_{2} \mathrm{SO}_{4}, \mathrm{HClO}_{4}$ and other affiliated chemicals were all obtained from Sinopharm Chemical Reagent. The $0.5 \%(\mathrm{w} / \mathrm{v})$ Nafion solution was prepared by $1: 10$ dilution with methanol of $5 \%(\mathrm{w} / \mathrm{v})$ Nafion solution (Sigma-Aldrich Co. LLC). The suspension of $0.5 \mathrm{mg} \mathrm{mL}^{-1}$ TiC nanoparticles (20 nm, Nanjing Emperor Nano Material Co., Ltd., China) were obtained by dispersing the powders with deionized water, and then ultrasonicated for $10 \mathrm{~min}$. All bottles and containers used for standards and samples were soaked in $5 \% \mathrm{HNO}_{3}$ at least for $24 \mathrm{~h}$ prior to use. Then they were thoroughly rinsed with deionized water and samples. All experiments were conducted at room temperature $\left(25^{\circ} \mathrm{C}\right)$ without the removal of oxygen from the solutions.

\subsection{Apparatus}

The morphologies of the modified glassy carbon electrode (GCE) were characterized by scanning electron microscopy (SEM, Hitachi S-4800, Japan) and transmission electron microscopy (TEM, Hitachi HT7700, Japan). Inductively coupled plasma-mass spectrometry (ICP-MS, ELAN DRCII, Perkin Elmer Instruments, USA) was used for comparative testing. Electrochemical Work Station (CHI 660D, Shanghai $\mathrm{CH}$ Instruments, Shanghai, China) was used throughout all the electrochemical experiments. A nano-TiC/Nafion coated glassy carbon electrode ( $3 \mathrm{~mm}$ in diameter) served as a working electrode, an $\mathrm{Ag} / \mathrm{AgCl}$ (saturated $\mathrm{KCl}$ solution) was used as a reference electrode, and a platinum foil counter electrode was employed as an auxiliary electrode. All potentials were measured with respect to the $\mathrm{Ag} / \mathrm{AgCl}$ reference electrode.

\subsection{Preparation of the modified GCEs}

The nano-TiC/Nafion coated GCE was prepared by adding $10 \mu \mathrm{L}$ nano-TiC $\left(0.5 \mathrm{mg} \mathrm{mL}^{-1}\right)$ on the surface of GCE and dried using an infrared lamp. Then $10 \mu \mathrm{L}$ Nafion $(0.5 \%, \mathrm{w} / \mathrm{v})$ was added on the surface of nano-TiC coated GCE and dried under the infrared lamp. Prior to use, the GCE was polished using aqueous alumina slurry $(0.3$ and $0.05 \mu \mathrm{m})$, washed with deionized water and acetone thoroughly, and then ultrasonicated in deionized water for $1 \mathrm{~min}$. For comparison, Nafion or nano-TiC coated GCE was prepared in the same manner, respectively.

\subsection{Preparation of real coastal water samples}

Water Samples were collected from Jiehe river and Xiaoqinghe river (two local coastal rivers, which flow into the Bohai Sea, Shandong province, China), and coastal water of the Bohai Sea. Samples were immediately filtered by $0.45 \mu \mathrm{m}$ membrane filters and collected in FEP bottles, then kept at $4{ }^{\circ} \mathrm{C}$ until determination. In order to remove the organic complexes, the samples were digested for $30 \mathrm{~min}$ at $\mathrm{pH}$ less than 2.0 in acid-cleaned quartz tubes using a 500 W UV lamp (Metrohm MVA-UV 705, Switzerland). Then the samples were diluted to an appropriate concentration and measured by the standard addition method.

\subsection{Electrochemical analysis procedure}

All experiments were performed in $0.1 \mathrm{M} \mathrm{HCl}$ electrolyte. The modified GCEs were electrochemically characterized by electrochemical impedance spectroscopy (EIS). EIS of modified electrodes was measured in $10 \mathrm{mM} \mathrm{K}_{3}\left[\mathrm{Fe}(\mathrm{CN})_{6}\right] / \mathrm{KCl}(0.1 \mathrm{M})$ solution with the frequency ranging from $10^{-2} \mathrm{~Hz}$ to $10^{5} \mathrm{~Hz}$. The cathodic responses of $\mathrm{Fe}^{3+}$ on the bare and modified GCEs were investigated by square wave voltammetry (SWV), using the following parameters: initial potential of $0.7 \mathrm{~V}$, final potential of $0.2 \mathrm{~V}$, an amplitude of $0.025 \mathrm{~V}$, increment potential of $0.001 \mathrm{~V}$, frequency of $15 \mathrm{~Hz}$, and a quiet time of $2 \mathrm{~s}$.

\section{Results and discussion}

\subsection{Characterization of the nano-TiC/Nafion modified electrode}

The surface morphologies of the various modified electrodes were characterized by SEM and TEM (Fig. 1). Compared with the smooth surface of the bare GCE (Fig. 1a), nano-TiC particles were compactly coated on the GCE surface with size distribution ranging from $20 \mathrm{~nm}$ to $60 \mathrm{~nm}$ (Fig. 1b). A thin membrane-like substance 

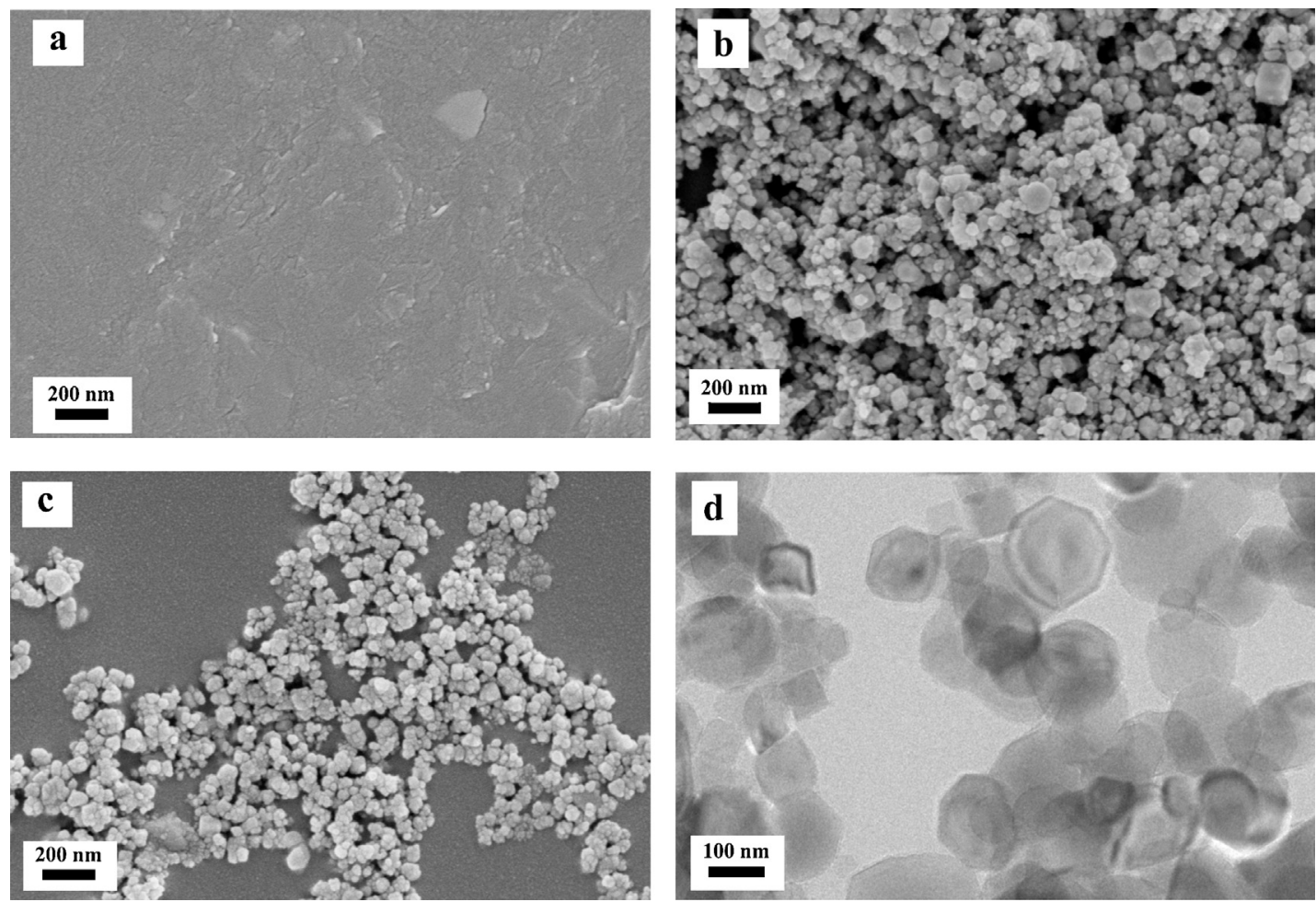

Fig. 1. SEM images of (a) bare GCE, (b) nano-TiC coated GCE, (c) nano-TiC/Nafion coated GCE, and (d) TEM image of nano-TiC.

was formed after Nafion coating of the nano-TiC coated GCE: some nano-TiC particles were incorporated into the Nafion film and others remained outside (Fig. 1c). TEM imaging showed that the nano-TiC is cubic in structure (Fig. 1d).

\subsection{Mechanism of $\mathrm{Fe}^{3+}$ reduction on fabricated sensor}

The sensing strategy is clearly illustrated in Scheme 1. Particles of nano-TiC which functioned as the electron transfer wires were well coated on the surface of a GCE to accelerate electron transfer rate and increase the current response since the reactions are limited by the rate of electron transfer. Then the "wire" surface was coated with Nafion to capture more $\mathrm{Fe}^{3+}$ by reaction (1), where $\mathrm{X}$ is the supporting electrolyte cation [26]. The detection process can be described by Eq. (2). During the voltammetric scan, $\mathrm{Fe}^{3+}$ is reduced to $\mathrm{Fe}^{2+}$, which is then re-oxidized chemically to $\mathrm{Fe}^{3+}$ by $\mathrm{H}_{2} \mathrm{O}_{2}$. The re-oxidized $\mathrm{Fe}^{3+}$ contributes again to the reduction current, causing a greatly improved sensitivity.

$$
\begin{gathered}
\begin{array}{c}
3\left(\mathrm{R}-\mathrm{SO}_{3}{ }^{-} \mathrm{X}^{+}\right)+\mathrm{Fe}^{3+} \leftrightarrows\left(\mathrm{R}-\mathrm{SO}_{3}{ }^{-}\right) \mathrm{Fe}^{3+}+3 \mathrm{X}^{+} \\
\left(\mathrm{R}-\mathrm{SO}_{3}{ }^{-}\right)_{3} \mathrm{Fe}^{3+}+\mathrm{e}^{-} \rightarrow\left(\mathrm{R}-\mathrm{SO}_{3}{ }^{-}\right)_{2} \mathrm{Fe}^{2+}
\end{array} \\
\mathrm{H}_{2} \mathrm{O}_{2}
\end{gathered}
$$

To verify the effect of nano-TiC and Nafion on $\mathrm{Fe}^{3+}$ detection, electrochemical impedance spectroscopy (EIS) was measured. Fig. 2 shows the Nyquist plots of the modified electrodes using $\left[\mathrm{Fe}(\mathrm{CN})_{6}\right]^{3-} /\left[\mathrm{Fe}(\mathrm{CN})_{6}\right]^{4-}$ redox couple as electrochemical probe. The squeezed semicircle at high frequency indicates an electrontransfer-limited process on an electroactive surface. This is followed by diffusion-controlled electron transfer, indicated by the linear plot region at low frequency. The Nyquist plot of nano-TiC coated GCE (Fig. 2b) showed a linear region only at low frequency, indicating that nano-TiC lowers the electron transfer resistance.

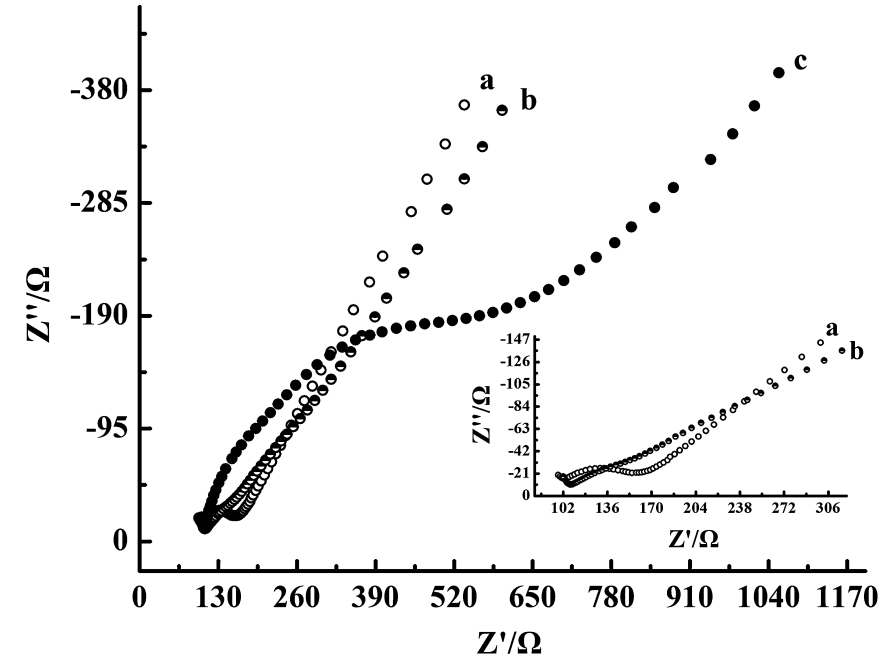

Fig. 2. Nyquist plots of bare GCE (curve a), nano-TiC coated GCE (curve b), and nano$\mathrm{TiC} /$ Nafion coated GCE (curve c) in $10 \mathrm{mM} \mathrm{K}_{3}\left[\mathrm{Fe}(\mathrm{CN})_{6}\right]$ and $0.1 \mathrm{M} \mathrm{KCl}$ solutions. Inset is enlarged drawing of the Nyquist plots of bare GCE (curve a), nano-TiC coated GCE (curve b) at higher frequency.

The electron transfer resistance (Rp) of the electroactive surface was measured by the semicircle diameter at high frequency. It was clearly observed that the Rp of nano-TiC/Nafion coated GCE (Fig. 2c) was significantly larger than that of bare GCE (Fig. 2a). Because Nafion is negatively charged, it will hinder electron transfer between $\left[\mathrm{Fe}(\mathrm{CN})_{6}\right]^{3-}$ and $\left[\mathrm{Fe}(\mathrm{CN})_{6}\right]^{4-}$. The results demonstrate that nano-TiC provides a larger electrode surface and more active sites as well as accelerating electron transfer, while Nafion facilitates a cation-exchange reaction for the preconcentration of $\mathrm{Fe}^{3+}$ To further understand the sensing mechanism, the effect of scan rate with the analytical signal of $\mathrm{Fe}^{3+}$ was studied by linear sweep voltammetry. The cathodic peak currents of $\mathrm{Fe}^{3+}$ increased linearly 


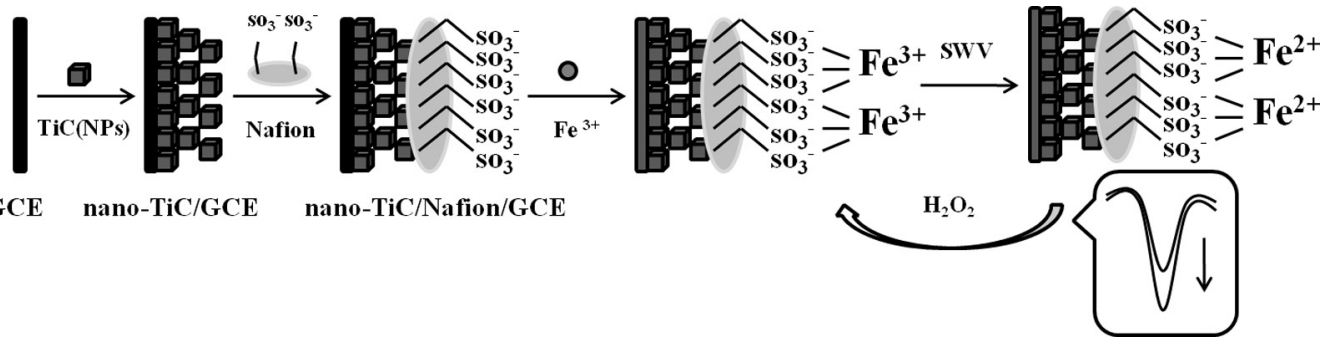

Scheme 1. Schematic illustration of the enhanced electrochemical detection strategy for $\mathrm{Fe}^{3+}$ on nano-TiC/Nafion modified electrode by catalytic stripping voltammetry.

with scan rates from $25 \mathrm{mV} \mathrm{s}^{-1}$ to $250 \mathrm{mV} \mathrm{s}^{-1}$. The regression equation is expressed as $i_{p}=-0.122 v-5.79\left(R^{2}=0.989\right)$. The results revealed that the electron transfer of $\mathrm{Fe}^{3+}$ with the nano-TiC/Nafion coated GCE was a typical surface-controlled electrochemical process [38]. Besides, to estimate the charge transfer coefficient $(\alpha)$, Laviron's model was used [39]. The plot of the cathodic peak potentials versus the logarithm of the scan rates yielded a straight line, the regression equation can be expressed as $E_{p}=-0.099 \log v+0.483$ $\left(R^{2}=0.989\right)$. From the slopes, $\alpha$ was estimated as 0.6 , indicating it was a quasi-reversible electron transfer process.

As shown in Fig. 3, the redox behaviors of $\mathrm{Fe}^{3+}$ at the fabricated sensor were studied by square wave voltammetry (SWV). No cathodic signal of $\mathrm{Fe}^{3+}$ was seen on the bare GCE (Fig. 3a and b). The SWV response for nano-TiC/Nafion coated GCE (Fig. 3h) was greatly larger than the responses for nano-TiC (Fig. 3d) and Nafion coated GCE (Fig. 3f). More importantly, the response current at the nano-TiC/Nafion modified electrode was larger than the sum of the currents at the nano-TiC and Nafion singly modified electrodes, indicating that nano-TiC combined with Nafion synergistically improve the cathodic current response of $\mathrm{Fe}^{3+}$. Since carbon nanomaterials can also provide the large surface area and considerable active sites, the responses of $\mathrm{Fe}^{3+}$ on multi-walled carbon nanotubes and graphene-modified GCE under the same experimental conditions were investigated. The results showed no obvious signal responses might be observed because there were large background currents at the potential range of $0.4-0.5 \mathrm{~V}$ caused by the oxygen-containing functional groups on the multi-walled carbon nanotubes and graphene's surfaces. But the background current was very low and smooth at nano-TiC modified electrode, which implies nano-TiC has significant advantages for $\mathrm{Fe}^{3+}$ detection than other carbon nanomaterials. Moreover, it has been demonstrated that the addition of $\mathrm{H}_{2} \mathrm{O}_{2}$ causes rapid oxidation of $\mathrm{Fe}^{2+}$ due to the generated radical species $\mathrm{HO}^{*}$, resulting in a large increase in the analytical signal. As shown in Fig. 4, the reduction peak for $\mathrm{Fe}^{3+}$ in the presence of $\mathrm{H}_{2} \mathrm{O}_{2}$ at the nano-TiC/Nafion coated GCE (Fig. 4c) has a straighter shape, and is about 3 times greater than the signal in the absence of $\mathrm{H}_{2} \mathrm{O}_{2}$ (Fig. 4b). These results indicate that the response current of $\mathrm{Fe}^{3+}$ can be obviously increased by synergistic and catalytic effect.

\subsection{Choice of supporting electrolyte}

The effect of supporting electrolyte to the response of $\mathrm{Fe}^{3+}$ was investigated because $\mathrm{Fe}^{3+}$ is easily hydrolyzed in slightly acidic and alkaline environments. Meanwhile, the supporting electrolyte can play a double role in the preconcentration of $\mathrm{Fe}^{3+}$ due to the complexation reactions by anions from the supporting electrolyte, and competition for the cation-exchange sites between the analyte and supporting electrolyte cation [20]. Therefore, $0.1 \mathrm{M}$ solutions of $\mathrm{H}_{2} \mathrm{SO}_{4}, \mathrm{HClO}_{4}, \mathrm{HNO}_{3}$, and $\mathrm{HCl}$ were chosen as possible electrolytes, the results showed that $\mathrm{HCl}$ solution was the best electrolyte for $\mathrm{Fe}^{3+}$ detection. The peak currents of $\mathrm{Fe}^{3+}$ in the different acidic media were increased in the order of $\mathrm{HClO}_{4}<\mathrm{H}_{2} \mathrm{SO}_{4}<\mathrm{HNO}_{3}<\mathrm{HCl}$, which was same as the previous report [22]. The peak current of $\mathrm{Fe}^{3+}$ in $\mathrm{HCl}$ was about ten times higher than other electrolytes. This can be attribute to the increasing acid anion adsorption on the surface of modified electrode and the reduction of $\mathrm{Fe}^{3+}$ to $\mathrm{Fe}^{2+}$ occurs fastest in $\mathrm{HCl}$ by judging the value of $\Delta \mathrm{E}_{\mathrm{p}}$ [22]. Besides, considering the reference electrode salt bridge and real coastal water samples with high salinity, choosing $\mathrm{HCl}$ as the electrolyte was more suitable.

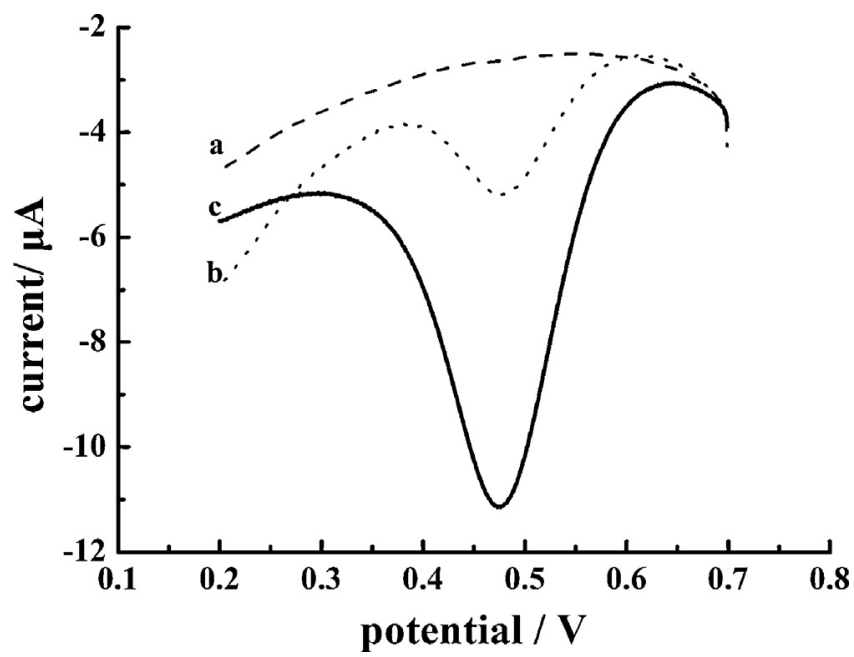

Fig. 4. Comparison of the SWV current signals at the nano-TiC/Nafion coated GCE in $0.1 \mathrm{M} \mathrm{HCl}$ containing $0 \mu \mathrm{M} \mathrm{Fe}^{3+}$ (curve a), $10 \mu \mathrm{M} \mathrm{Fe}^{3+}$ (curve b), $10 \mu \mathrm{M} \mathrm{Fe}^{3+}$ and $20 \mathrm{mM} \mathrm{H}_{2} \mathrm{O}_{2}$ (curve $\mathrm{c}$ ).
Fig. 3. SWVs of bare GCE (curve a and b), nano-TiC coated GCE (curve $c$ and d), Nafion coated GCE (curve e and f), and nano-TiC/Nafion coated GCE (curve $g$ and $h$ ) in $0.1 \mathrm{M} \mathrm{HCl}$ without (curve a, c, e, g) and with (curve b, d, f, h) $10 \mu \mathrm{M} \mathrm{Fe}^{3+}$.

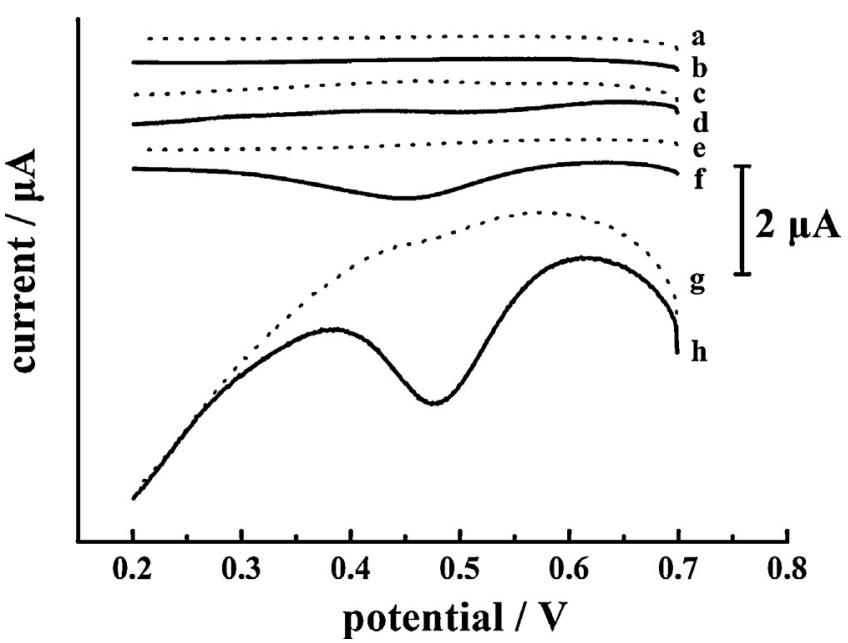




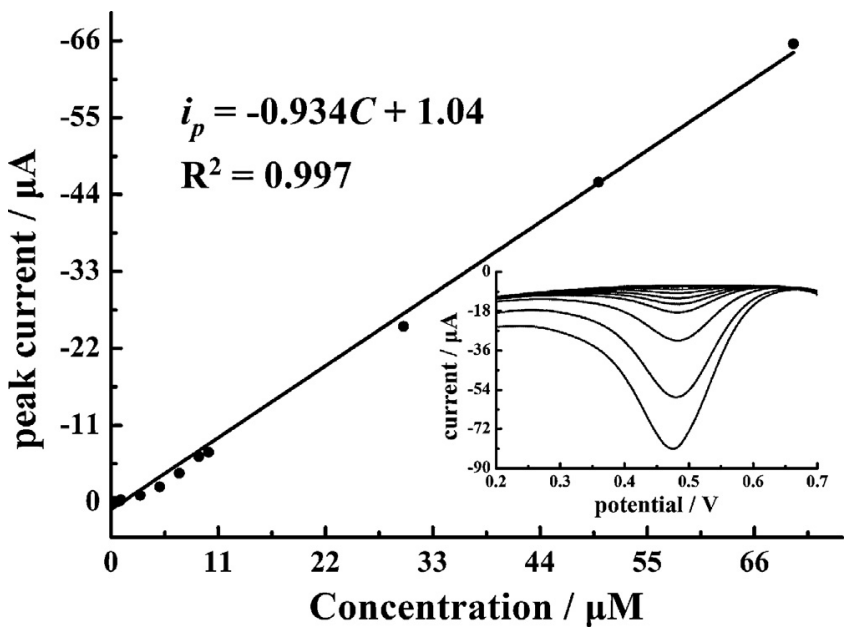

Fig. 5. Calibration curve of $\mathrm{Fe}^{3+}$ on the nano-TiC/Nafion coated GCE in $0.1 \mathrm{M} \mathrm{HCl}$ in the presence of $20 \mathrm{mM} \mathrm{H}_{2} \mathrm{O}_{2}$. Inset is the current response of $\mathrm{Fe}^{3+}$ concentrations in the linear range from $0.07 \mu \mathrm{M}$ to $70 \mu \mathrm{M}$.

\subsection{Calibration curve}

For accurate quantitative analysis, a linear calibration curve between cathodic current and $\mathrm{Fe}^{3+}$ concentration is necessary. As shown in Fig. 5, the current was linearly related to the concentration of $\mathrm{Fe}^{3+}$ under the concentration range of $0.07-70 \mu \mathrm{M}$. The linear regression equation was $i_{p}=-0.934 C+1.04\left(R^{2}=0.997\right)$. The sensitivity and detection limit of the nano-TiC/Nafion coated GCE for $\mathrm{Fe}^{3+}$ determination were $0.93 \mu \mathrm{A} \mu \mathrm{M}^{-1}$ and $7.2 \mathrm{nM}(\mathrm{s} / \mathrm{n}=3)$, respectively. These results indicate that the proposed modified GCE can qualitatively and quantitatively detect $\mathrm{Fe}^{3+}$ in experimental system. Additionally, comparisons about iron determination by this sensor and other Nafion modified electrodes were presented in Table 1. Our new fabricated sensor shows better sensitivity, lower detection limit for $\mathrm{Fe}^{3+}$, and easier experimental processes compared with other Nafion modified electrodes.

\subsection{Reproducibility, repeatibility and selectivity}

The inter-sensor reproducibility of the nano-TiC/Nafion modified electrode was evaluated in $10 \mu \mathrm{M} \mathrm{Fe}^{3+}$ by six independently sensors prepared in the same way and the corresponding relative standard deviation (RSD) was $4.7 \%$. The repeatability of the modified sensor was studied by detecting $10 \mu \mathrm{M} \mathrm{Fe}^{3+}$ using the same sensor for six measurements and the RSD was $2.8 \%$, which indicated there were no significant differences among the analytical signals. To verify whether there were cathodic responses to other cations, various foreign species were added into $0.1 \mathrm{M} \mathrm{HCl}$ containing $10 \mu \mathrm{M} \mathrm{Fe}^{3+}$. Additions of 30 -fold $\mathrm{Cu}^{2+}, \mathrm{Cr}^{3+}$, and 10 -fold $\mathrm{Zn}^{2+}$ and $\mathrm{As}^{3+}$ did not affect the determination of $\mathrm{Fe}^{3+}$ (5\% current change). The addition of less than $0.03 \mathrm{M}$ sodium chloride also did not influence the signal of $\mathrm{Fe}^{3+}$. However, adding equivalent $\mathrm{Ag}^{+}$resulted in

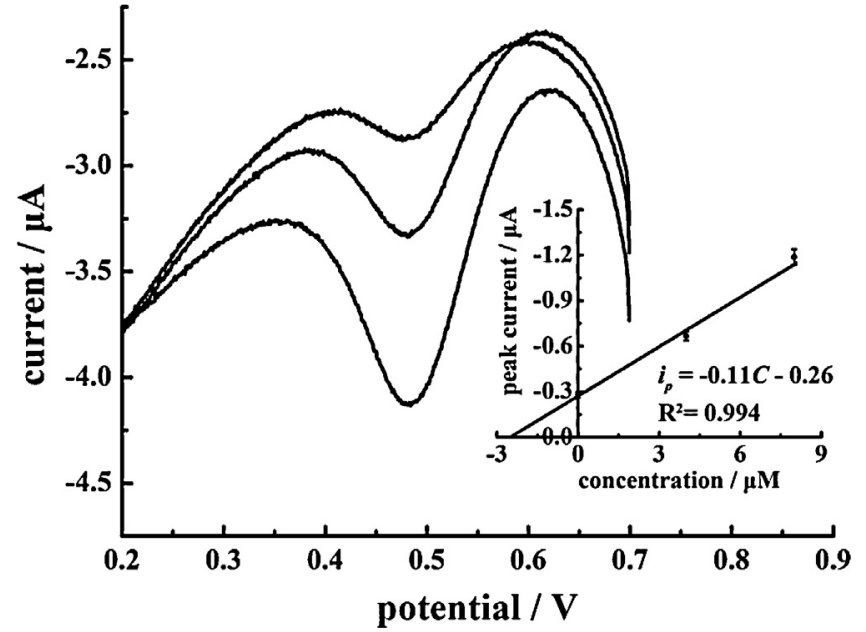

Fig. 6. Typical voltammograms for the determination of $\mathrm{Fe}^{3+}$ in water samples from the Jiehe River by the standard addition method. The concentrations of $\mathrm{Fe}^{3+}$ are 0 4 and $8 \mu \mathrm{M} \mathrm{Fe}^{3+}$ (from top to bottom). The inset shows the corresponding curve $(n=3)$.

a sharp decrease in signal because of silver chloride precipitation on the electrode surface, which hinders electron transfer between the electrode and $\mathrm{Fe}^{3+}$ [41]. The influence of common reducible organic species such as humic substances and catechol were also investigated. The results showed that the addition of 20 -fold humic substances did not interfere with the signals, but adding 5-fold catechol would decrease the peak current and change the peak shape due to catechol was one of the common chelators of iron. However, after the UV-digestion, the peak currents returned to the original values because all organic substances in solution have been destroyed during UV digestion. So, this method was not obviously interfered with the organic species after UV digestion.

\subsection{Practical application}

After having demonstrated the selectivity and sensitivity of the nano-TiC/Nafion coated GCE for $\mathrm{Fe}^{3+}$ in optimal experimental conditions, it has been applied to determine the total dissolved iron in real coastal waters. All water samples were filtered with $0.45 \mu \mathrm{m}$ membrane filters and then UV-digested after adjusting the $\mathrm{pH}$ to less than 2.0 to make sure all ligands bound to iron were released, and all iron species were oxidized to $\mathrm{Fe}^{3+}$. SWVs were obtained under optimal conditions for water samples from the Jiehe river (Fig. 6). The water sample was diluted 10 times, then added to $0.1 \mathrm{M} \mathrm{HCl}$ for detection. A series of SWVs were obtained after the successive addition of 0,4 , and $8 \mu \mathrm{M} \mathrm{Fe}^{3+}$, and the linear regression is shown in the inset of Fig. 6. The concentration of total dissolved iron in the Jiehe river was determined to be $2.3 \mu \mathrm{M}$, consistent with the ICP-MS result $(2.4 \mu \mathrm{M})$. A comparison of results on the total dissolved iron in coastal waters from our proposed method and ICP-MS are shown in Table 2. The results show that the

Table 1

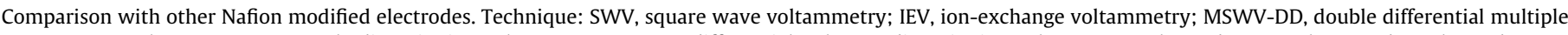

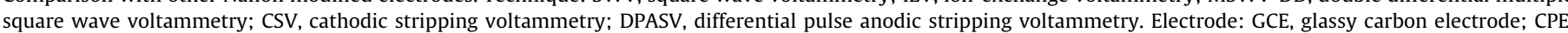
carbon paste electrode.

\begin{tabular}{|c|c|c|c|c|c|c|}
\hline Technique & Electrode & Modifier/Complexing agent (if any) & Speciation & LOD (M) & Year & Reference \\
\hline IEV & GCE & Nafion, Tosflex & $\mathrm{Fe}^{3+}+\mathrm{Fe}^{2+}$ & $1.0 \times 10^{-7}$ & 2002 & [17] \\
\hline MSWV-DD & GCE & Nafion & $\mathrm{Fe}^{3+}+\mathrm{Fe}^{2+}$ & $1.5 \times 10^{-6}$ & 2001 & [16] \\
\hline CSV & GCE & Nafion, $\alpha \alpha$-bipyridine & $\mathrm{Fe}^{3+}$ & $8.9 \times 10^{-10}$ & 1995 & [18] \\
\hline DPASV & CPE & 2,2-bipyridyl, Nafion & $\mathrm{Fe}^{2+}$ & $1.0 \times 10^{-8}$ & 1991 & [40] \\
\hline DPASV & $\mathrm{CPE}$ & Nafion, 1,10-phenanthroline & $\mathrm{Fe}^{2+}$ & $3.0 \times 10^{-8}$ & 1990 & [19] \\
\hline SWV & GCE & nano-TiC and Nafion & $\mathrm{Fe}^{3+}$ & $7.2 \times 10^{-9}$ & 2015 & This work \\
\hline
\end{tabular}


Table 2

Comparison of nano-TiC/Nafion coated GCE and ICP-MS for determination of total dissolved iron in coastal waters.

\begin{tabular}{lcc}
\hline Samples & $\begin{array}{l}\text { Detected by } \\
\text { this method } \\
(\mu \mathrm{M})\end{array}$ & $\begin{array}{c}\text { Detected by } \\
\text { ICP-MS }(\mu \mathrm{M})\end{array}$ \\
\hline Coastal Jiehe river water 1 & $2.3 \pm 0.2$ & 2.4 \\
Coastal Jiehe river water 2 & $12.0 \pm 0.2$ & 11.9 \\
Coastal Xiaoqinghe river water 3 & $4.3 \pm 0.2$ & 4.3 \\
Coastal sea water & $4.4 \pm 0.2$ & 4.5 \\
\hline
\end{tabular}

proposed method is suitable for the total dissolved iron determination for real coastal water samples.

\section{Conclusions}

In summary, we have developed a novel nano-TiC/Nafion coated GCE that exhibits high selectivity and sensitivity for $\mathrm{Fe}^{3+}$ determination in real coastal water samples without using complexing agents. Our new modified GCE shows better sensitivity, lower detection limit for $\mathrm{Fe}^{3+}$ detection. This catalytic stripping method has easier experimental processes and is suitable for total dissolved iron determination in coastal water samples. The nano-TiC/Nafion coated GCE will be of great benefit to investigate the effects of iron in coastal aquatic biogeochemistry.

\section{Acknowledgments}

This work was financially supported by the National Natural Science Foundation of China (41276093), the Youth Innovation Promotion Association (2011170), and the Outstanding Young Scientists Program of CAS.

\section{References}

[1] W.G. Sunda, S.A. Huntsman, Iron uptake and growth limitation in oceanic and coastal phytoplankton, Mar. Chem. 50 (1995) 189-206.

[2] J.H. Martin, R.M. Gordon, S. Fitzwater, W.W. Broenkow, VERTEX: phytoplankton/iron studies in the Gulf of Alaska, Deep-Sea Res. A 36 (1989) 649-680.

[3] P.J. Statham, Y. Jacobson, C.M.G. van den Berg, The measurement of organically complexed $\mathrm{Fe}(\mathrm{II})$ in natural waters using competitive ligand reverse titration, Anal. Chim. Acta 743 (2012) 111-116.

[4] C. Schlosser, J.K. Klar, B.D. Wake, J.T. Snow, D.J. Honey, E.M.S. Woodward, M.C. Lohan, E.P. Achterberg, C.M. Moore, Seasonal ITCZ migration dynamically controls the location of the (sub) tropical Atlantic biogeochemical divide, Proc. Natl. Acad. Sci. U. S. A. 111 (2014) 1438-1442.

[5] K.W. Bruland, E.L. Rue, The Biogeochemistry of Iron in Seawater IUPAC Series on Analytical and Physical Chemistry of Environmental Systems, John Wiley, New York, 2001, pp. 255-290.

[6] D.G. Capone, D.A. Hutchins, Microbial biogeochemistry of coastal upwelling regimes in a changing ocean, Nat. Geosci. 6 (2013) 711-717.

[7] Z. Chase, K.S. Johnson, V.A. Elrod, J.N. Plant, S.E. Fitzwater, L. Pickell, C.M. Sakamoto, Manganese and iron distributions off central California influenced by upwelling and shelf width, Mar. Chem. 95 (2005) 235-254.

[8] S.M. Zhou, C.X. Zhang, The main characteristic parameters and chemical environment of the red tides, J. Oceanogr. Huanghai Bohai Seas 10 (1992) 73-76 (in Chinese).

[9] E.P. Achterberg, T.W. Holland, A.R. Bowie, R.F.C. Mantoura, P.J. Worsfold, Determination of iron in seawater, Anal. Chim. Acta 442 (2001) 1-14.

[10] D. Pan, Y. Wang, Z. Chen, T. Lou, W. Qin, Nanomaterial/ionophore-based electrode for anodic stripping voltammetric determination of lead: an electrochemical sensing platform toward heavy metals, Anal. Chem. 81 (2009) 5088-5094

[11] L.M. Laglera, J. Santos-Echeandía, S. Caprara, D. Monticelli, Quantification of iron in seawater at the low picomolar range based on optimization of bromate/ammonia/dihydroxynaphtalene system by catalytic adsorptive cathodic stripping voltammetry, Anal. Chem. 85 (2013) 2486-2492.

[12] C.M.G. van den Berg, Chemical speciation of iron in seawater by cathodic stripping voltammetry with dihydroxynaphthalene, Anal. Chem. 78 (2006) 56-163.

[13] J. Wang, Stripping analysis at bismuth electrodes: a review, Electroanalysis 17 (2005) 1341-1346.

[14] M.E. Dollhopf, K.H. Nealson, D.M. Simon, G.W. Luther, Kinetics of Fe (III) and Mn (IV) reduction by the Black Sea strain of Shewanella putrefaciens using in situ solid state voltammetric Au/Hg electrodes, Mar. Chem. 70 (2000) 171-180.
[15] M.H. Mashhadizadeh, I.S. Shoaei, N. Monadi, A novel ion selective membrane potentiometric sensor for direct determination of Fe (III) in the presence of Fe (II), Talanta 64 (2004) 1048-1052.

[16] P. Ugo, L.M. Moretto, D. Rudello, E. Birriel, J. Chevalet, Trace iron determination by cyclic and multiple square-wave voltammetry at Nafion coated electrodes. Application to pore-water analysis, Electroanalysis 13 (2001) 661-668.

[17] P. Ugo, L.M. Moretto, A. De Boni, P. Scopece, G.A. Mazzocchin, Iron (II) and iron (III) determination by potentiometry and ion-exchange voltammetry at ionomer-coated electrodes, Anal. Chim. Acta 474 (2002) 147-160.

[18] S.X. Yang, L.H. Du, B.X. Ye, Cathodic stripping voltammetry determination of trace iron with a chemically modified electrode, Microchem. J. 52 (1995) 216-222.

[19] Z.Q. Gao, P. Li, G. Wang, Z. Zhao, Preconcentration and differential-pulse voltammetric determination of iron(II) with Nafion-1,10-phenanthroline-modified carbon paste electrodes, Anal. Chim. Acta 241 (1990) 137-146.

[20] E.M. Ghoneim, Simultaneous determination of Mn (II), Cu (II) and Fe (III) as 2-(5'-bromo-2'-pyridylazo)-5-diethylaminophenol complexes by adsorptive cathodic stripping voltammetry at a carbon paste electrode, Talanta 82 (2010) 646-652.

[21] R. Segura, M.I. Toral, V. Arancibia, Determination of iron in water samples by adsorptive stripping voltammetry with a bismuth film electrode in the presence of 1-(2-piridylazo)-2-naphthol, Talanta 75 (2008) 973-977.

[22] E. Zakharova, E. Elesova, G. Noskova, M. Lu, R. Compton, Direct voltammetric determination of total iron with a gold microelectrode ensemble, Electroanalysis 24 (2012) 2061-2069.

[23] R.K. Shervedani, A. Hatefi-Mehrjardi, A. Asadi-Farsani, Sensitive determination of iron (III) by gold electrode modified with 2-mercaptosuccinic acid selfassembled monolayer, Anal. Chim. Acta 601 (2007) 164-171.

[24] M.W. Espenscheid, A.R. Ghatak-Roy, R.B. Moore, R.M. Penner, M.N. Szentirmay, C.R. Martin, Sensors from polymer modified electrodes, J. Chem. Soc. Faraday Trans. I 82 (1986) 1051-1070.

[25] P. Ugo, L.M. Moretto, Ion-exchange voltammetry at polymer-coated electrodes: principles and analytical prospects, Electroanalysis 7 (1995) 1105-1113.

[26] N. Zhou, H. Chen, J. Li, L. Chen, Highly sensitive and selective voltammetric detection of mercury (II) using an ITO electrode modified with 5-methyl-2thiouracil, graphene oxide and gold nanoparticles, Microchim. Acta 180 (2013) 493-499.

[27] C.S. Shan, H.F. Yang, J.F. Song, D.X. Han, A. Ivaska, L. Niu, Direct electrochemistry of glucose oxidase and biosensing for glucose based on graphene, Anal. Chem. 81 (2009) 2378-2382.

[28] M.B. Gholivand, B. Geravandi, M.H. Parvin, Anodic stripping voltammetric determination of iron (II) at a carbon paste electrode modified with dithiodianiline (DTDA) and gold nanoparticles (GNP), Electroanalysis 23 (2011) 1345-1351.

[29] D.I. Anguiano, M.G. Garcia, C. Ruiz, J. Torres, I. Alonso-Lemus, L. AlvarezContreras, Y. Verde-Gomez, E. Bustos, Electrochemical detection of iron in a lixiviant solution of polluted soil using a modified glassy carbon electrode, Int J. Electrochem. 739408 (2012) 6.

[30] M. Lin, H. Han, D. Pan, H. Zhang, Z. Su, Voltammetric determination of tota dissolved iron in coastal waters using a glassy carbon electrode modified with reduced graphene oxide, Methylene Blue and gold nanoparticles, Microchim. Acta 182 (2015) 805-813.

[31] T. Yu, YH. Deng L. Wang R. Liu, LJ. Zhang B. Tu, D. . Zhao, Ordered mesoporous nanocrystalline titanium-carbide/carbon composites from in situ carbothermal reduction, Adv. Mater. 19 (2007) 2301-2306.

[32] D.W. Flaherty, R.A. May, S.P. Berglund, K.J. Stevenson, C.B. Mullins, Low temperature synthesis and characterization of nanocrystalline titanium carbide with tunable porous architectures, Chem. Mater. 22 (2010) 319-329.

[33] J.A. Rodríguez, L. Feria, T. Jirsak, Y. Takahashi, K. Nakamura, F. Illas, Role of Au-C interactions on the catalytic activity of Au nanoparticles supported on $\mathrm{TiC}(001)$ toward molecular oxygen dissociation, J. Am. Chem. Soc. 132 (2010) 3177-3186.

[34] M. Wang, O. Sheng, D. Zhang, Y. He, J. Zheng, TiC nanoparticles-chitosan composite film for the direct electron transfer of myoglobin and its application in biosensing, Bioelectrochemistry 86 (2012) 46-53.

[35] Y.H. Chang, C.W. Chiu, Y.C. Chen, C.C. Wu, C.P. Tsai, J.L. Wang, H.T. Chiu, Syntheses of nano-sized cubic phase early transition metal carbides from metal chlorides and n-butyllithium, J. Mater. Chem. 12 (2002) 2189-2191.

[36] Y. Bi, H. Hu, S. Ouyang, G. Lu, J. Cao, J. Ye, Photocatalytic and photoelectric properties of cubic $\mathrm{Ag}_{3} \mathrm{PO}_{4}$ sub-microcrystals with sharp corners and edges, Chem. Commun. 48 (2012) 3748-3750.

[37] M. Jin, H. Zhang, Z. Xie, Y. Xia, Palladium nanocrystals enclosed by $\{100\}$ and $\{111\}$ facets in controlled proportions and their catalytic activities for formic acid oxidation, Energy Environ. Sci. 5 (2012) 6352-6357.

[38] S.F. Wang, T. Chen, Z.L. Zhang, X.C. Shen, Z.X. Lu, D.W. Pang, K. Wong, Direct electrochemistry and electrocatalysis of heme proteins entrapped in agarose hydrogel films in room-temperature ionic liquids, Langmuir 21 (2005) 9260-9266.

[39] E. Laviron, General expression of the linear potential sweep voltammogram in the case of diffusionless electrochemical systems, J. Electroanal. Chem. 101 (1979) 19-28.

[40] Z. Gao, P. Li, Z. Zhao, Determination of iron (II) with chemically-modified carbon-paste electrodes, Talanta 38 (1991) 1177-1184.

[41] X. Chu, X. Fu, K. Chen, G.L. Shen, R.Q. Yu, An electrochemical stripping met alloimmunoassay based on silver-enhanced gold nanoparticle label, Biosens. Bioelectron. 20 (2005) 1805-1812. 


\section{Biographies}

Mingyue Lin earned the BS degree of environmental engineering in 2012 at Nanjing University of Information Science \& Technology. She is currently a Ph.D. student at Yantai Institute of Coastal Zone Research, Chinese Academy of Sciences. Her scientific interest includes electrochemical sensors, analysis of environmental pollutants and nutrients.

Dawei Pan is currently a professor at Yantai Institute of Coastal Zone Research, Chinese Academy of Sciences. He received the Ph.D. degree in 2007 at Hunan University. His research interests focus on electrochemical sensor system's research and integration, electroanalysis of coastal environment's pollutants and nutrients, and interface electrochemistry.
Xueping Hu earned the BS degree in 2013 at Linyi University. She is currently a master's degree student at Yantai Institute of Coastal Zone Research, Chinese Academy of Sciences. Now she is engaged in on-spot detection of pollution by screen-printed electrode.

Haitao Han is currently a research assistant at Yantai Institute of Coastal Zone Research, Chinese Academy of Sciences. He earned his master degree in 2014 at Yantai University. His research interests are in the area of synthesis of novel nanocomposites, electrochemical sensors and environmental analysis.

Fei Li earned her BS degree in 2013 at Shandong Normal University. She is currently a master's degree student at college of Chemical Engineering and Materials Science, Shandong Normal University. Her scientific interests focus on electrochemical sensors and microelectrode. 